\title{
A Study on Virtual Roaming System of Campus Panorama Based on VR
}

\author{
Wei Xiao \\ Ganzhou Teachers College, 341000, China
}

Keywords: VR, Campus Panorama, Virtual Roaming System.

\begin{abstract}
With the continuous development of modern science and technology, the introduction of VR technology has provided new support for the construction and management of campus in the future. This paper takes the virtual roaming technology of campus panorama based on VR technology as the discussion point, after discussing the future application and development characteristics of VR, analyzes the production flow and key technology of VR technology, and focuses on the modern science and technology such as panoramic shooting technology, image analysis technology and so on.
\end{abstract}

\section{Basic Features and Related Definitions of VR Panorama Technology}

Under the background of the construction of Internet +, modern VR technology has become the representative of the new development concept, influenced by the new educational situation, and the new application based on the development of VR technology is being widely accepted. Compared with the traditional online management, VR has become an important driving force to promote the rapid development of campus construction, enrollment propaganda, intelligent campus, virtual library and other technologies in the future. As shown in figure 1, in the case of VR virtual roaming management system, the "air-to-air" management model has been established, and no new ideas have been provided for the future construction and development of the campus.

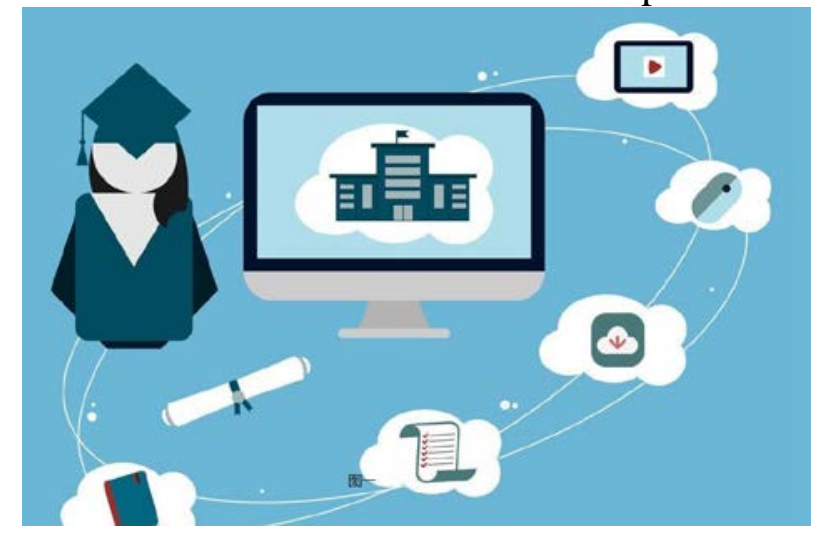

Figure 1 Campus panorama

The development of modern technology provides a platform for the growth of VR technology, compared with the previous network information import, online information application and other technologies, VR emphasizes the "real-time, interactive" information, compared with the thin information display, picture display, text display and other display methods, VR can create an experience environment and interactive environment for the individual experience, and introduce a stronger "sense of reality" for the corresponding object.[1]. Under the new network background, the VR technology can establish the corresponding experience and manipulation environment for the relevant personnel, so as to maximize the retention of the user's sense of reality.

VR panoramic technology is a scientific display technology developed in the context of the Internet. Compared with the traditional online display mode, VR panoramic technology is based on the virtualization technology of panoramic real images. In the process of establishing the virtual roaming management system, the relevant technicians need to carry on the omni-directional and multi-line continuous shooting to the campus, and use the information technology to splice the 
image and video, and use the final image to carry on the corresponding suture display. Compared with the online display mode, the virtual roaming system based on VR technology emphasizes the "visualization" and "vivid" of landscape display, and optimizes the corresponding blind area of vision. Compared with the traditional management concept, the display ability of virtual roaming system based on VR technology is more comprehensive.

\section{Application Advantage of Virtual Roaming System}

The traditional campus display model focuses on the "iconic buildings" and "iconic" characters inside the school. Under this display concept, the related display work is inclined to plane display, picture display, visitors can not get available knowledge in a short time, and visit blind spots, construction blind spots, management blind spots and other problems still exist[2]Under the virtual roaming system, visitors can initiate $360^{\circ}$ or even $720^{\circ}$ of visits, change the virtual environment by looking down and looking up, and import the corresponding real-time interactive activities. Under the virtual roaming system, visitors can initiate interactive activities based on changing focal length, clicking on virtual objects and so on.

Compared with the traditional one-sided display mode, the VR panoramic roaming virtual system allows the object to complete three-dimensional display and real-time interaction activities on a variety of terminals. When launching the visit, the visiting individual can use modern technology products such as computer, tablet computer, mobile phone to participate in the corresponding virtual visit activities, and should complete the corresponding display task in a more novel and faster way.

\section{Production Flow and Core Technology of Panoramic Virtual Roaming Technology Based on VR}

In the process of making panorama at the present stage, the production flow of panorama is mainly divided into four parts: panorama shooting, image stitching, image stitching and panoramic display. In the process of applying panoramic roaming virtual technology, relevant technicians should not only complete the introduction of related picture technology in time, but also use network technology to import corresponding graphic resources, text explanation resources, and actually solve the basic problems such as color difference and background.

\subsection{Panorama Shoot}

In a literal sense, the panorama means "a picture containing all the features of the scene taken ". As shown in figure 2, when the volume is the same and the resources are consumed, the panorama can complete the integration and combing of a large number of scenery resources in a short time, and help visitors to master the basic characteristics of the relevant scenery in a short time.

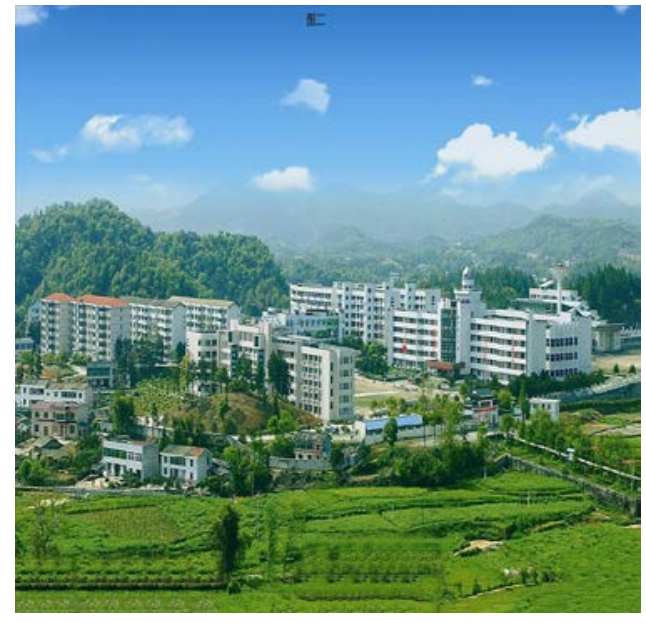

Figure 2 Campus panorama 
Under the limitation of virtual roaming system, the production of panorama can get more abundant technical support: the old panorama shooting technology is mainly photo-based, and the second phase processing of panorama is completed by the way of picture splicing and later art editing, but limited by the technical level, the panorama produced under this shooting technology can not fully meet the requirements of later application; At the present stage of panoramic photography, the technology of shooting and network processing has been greatly improved. At present, the camera used in related activities already has the ability of panoramic students to succeed. On the premise of retaining the basic photography technology, it can fully reflect the simple and fast characteristics of modern photography technology, but it still has the problems of high equipment cost and difficult application. At present, the more common panoramic shooting equipment has Nokia OZO、GoPro, etc. For some units that need to take panoramic pictures but lack relevant equipment, they will choose ordinary digital cameras and stitch the photos after taking multiple scene photos. Compared with the high cost shooting mode, this application mode is more difficult to operate, but can greatly control the cost. The common photography equipment has digital camera cloud head, PS and other processing tools.

\subsection{Image Mosaic}

For the application unit which lacks the panoramic shooting equipment, when it produces the intention to apply the panoramic picture and lacks the specialized equipment, it is generally necessary to introduce the image stitching and stitching technology[3]. In the actual shooting process, different images are affected by the shooting environment, shooting technology and other factors. Through the splicing and fusion processing of images, professional technicians can standardize the difference items such as image calming, perspective discoloration, color aberration, rotation, etc., while eliminating the suture image while preserving the high visibility and application value of the image to achieve the natural transition of the image. Compared with the panoramic shooting technology of "one time molding ", image splicing emphasizes the organic fusion between different pictures, and it pays attention to the coordination and unity of the whole shooting environment and the application environment.

\subsection{Image Stitching}

Image stitching is a picture processing technology based on the requirements of picture application. Compared with picture stitching, image stitching focuses on the optimization and fusion of pictures, and its work focuses on "using modern technology to complete the integration of multiple pictures ". Under the processing of image stitching technology, panoramic picture can highlight the key points of display, and show the precision and refinement of panoramic picture.

After completing the splicing task, the relevant technicians need to import the corresponding suture processing technology, in this link need to carry on the advanced processing to the image according to the panoramic picture application environment, the application request, as shown in figure 3, such as using the network tool to remove the picture splicing crack, eliminate the bad shadow in the picture, repair the corresponding parts, etc., rely on the organic cooperation of multiple technologies, each independent picture can generate a more natural panorama. It is worth mentioning that with the continuous development of modern image processing technology, image stitching technology has become an important part of panoramic image processing activities, through the beautification, optimization, panorama can more comprehensive display of their own image display characteristics. 


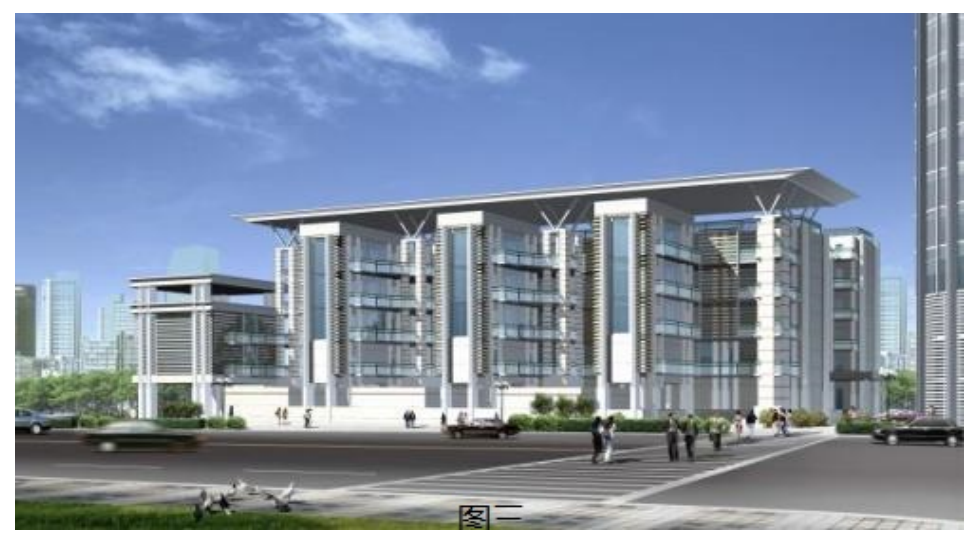

Figure 3 Campus panorama

\subsection{Panorama Display}

The panoramic display can make the visitors experience the real scene in a short period of time and get the interactive feeling. In the past economic activities, the panoramic display is generally applied to the e-commerce activities, such as the real estate display online, the tourism virtual route planning, the development of virtual education activities and so on. During the research activities of virtual roaming system based on VR, the panorama can retain the authenticity of the related display activities, enhance the visitors' sense of capture, and realize the simplification of user operation and the sublimation of user experience.

On the basis of the school's comprehensive configuration system and the difference of hardware configuration level, different display measures are often adopted in the panoramic display activities at the present stage — For the low level of comprehensive configuration, the general choice of popular computer, LED screen and other ways to complete the display work, relying on a variety of one-to-one configuration, it can retain the authenticity and dissemination of the relevant display work; On the one hand, the panoramic view is based on the development of modern information technology. After re-introduction into the network, the related promotion work will not be hindered by information technology. On the other hand, the excellent panoramic view display work can open the available information in time, enhance the competitiveness of the outside world, and improve the quality and dissemination efficiency of the panoramic view through mobile phone, notebook computer, personal information equipment and so on.

\section{Conclusion}

Based on the VR processing technology, the emergence of the campus panoramic virtual roaming system provides new thinking for the subsequent campus display and campus management. In the process of constructing the virtual roaming system, relevant workers should do a good job of image shooting and processing from the two angles of image processing technology and image application environment.

\section{Acknowledgements}

Research on Application of virtual reality technology based on Panorama-—Take Ganzhou Teachers College as an example (Science and technology research project of Jiangxi Provincial Department of education in 2019, Project No: (GJJ191252).

\section{References}

[1] Zheng, Wei. Research on Virtual Roaming System of Campus Panorama Based on VR. Digital World, no.12, pp. 550-550, 2017.

[2] Ma, Cheng., Yan, Junjun., Xu, Jian. Research and Construction of Virtual Campus Roaming 
System Based on VR. Software, vol. 40, no. 03, pp. 112-120, 2019.

[3] Ding, Xuejing. The Design and Implementation of Virtual Campus Roaming System Based on VR. Journal of Anqing Normal University (Natural Science Edition), no. 01, pp. 52-56, 2014. 